\title{
Crystallization Kinetics of iPP: Influence of Operating Conditions and Molecular Parameters
}

\author{
V. La Carrubba, S. Piccarolo, V. Brucato \\ Dipartimento di Ingegneria Chimica dei Processi e dei Materiali, Università di Palermo, Viale delle Scienze, \\ 90128 Palermo, Italy
}

Received 5 September 2006; accepted 21 November 2006

DOI 10.1002/app.25871

Published online in Wiley InterScience (www.interscience.wiley.com).

\begin{abstract}
An analysis of the crystallization kinetics of different grades of isotactic polypropylene (iPP) is here presented. To describe the crystallization kinetics as a function of molecular and operating parameters, the methodological path followed was the preparation of quenched samples of known cooling histories, calorimetric crystallization isotherms tests, differential scanning calorimetry cooling ramps, wide angle X-ray diffraction (WAXD) measurements, and density determination. The WAXD analysis performed on the quenched iPP samples confirmed that during the fast cooling at least a crystalline structure and a mesomorphic one form. The diffractograms were analyzed by a deconvolution procedure, to identify the relationship between the cooling history and the distribution of
\end{abstract}

the crystalline phases. The whole body of results (including calorimetric ones) provides a wide basis for the identification of a crystallization model suitable to describe solidification in polymer-processing operations, based on the KolmogoroffAvrami-Evans nonisothermal approach. The kinetic parameters, determined for all the materials, are discussed, highlighting the effect of molecular parameters on the crystallization kinetics: molecular mass and distribution, tacticity, nucleating agents, and ethylene units content. (c) 2007 Wiley Periodicals, Inc. J Appl Polym Sci 104: 1358-1367, 2007

Key words: WAXS; density; crystallization; polyolefins; processing

\section{INTRODUCTION}

The crystalline structure of the polymer is extremely relevant for practical purposes as it significantly influences the properties of final products. Generally speaking, polymer crystallization under processing conditions cannot be strictly considered an "equilibrium" phenomenon, since it is not possible to separate thermodynamics from kinetic effects on processes. Furthermore, polymeric materials crystallization is always limited by molecular mobility, and very often leads to metastable phases. ${ }^{1}$ Evidences of the formation of metastable phases under drastic conditions (high cooling rates and/or high deformation rates) have been widely reported for several semicrystalline polymers. $^{2-6}$

From the aforementioned arguments, it comes out that the structure of semicrystalline polymers, being kinetically controlled, is always strongly affected by processing conditions. In actual industrial polymer processing, crystallization generally occurs under

Correspondence to: V. La Carrubba (lacarrubba@dicpm. unipa.it).

Contract grant sponsor: EU Brite; contract grant number: BRPR.CT96.0147.

Contract grant sponsor: The Italian Government MURST Funds (40\% Quota).

Journal of Applied Polymer Science, Vol. 104, 1358-1367 (2007) (C)2007 Wiley Periodicals, Inc. nonisothermal conditions and analysis of crystallization kinetics is not straightforward.

With reference to isotactic polypropylene (iPP), it is well known that crystallization toward monoclinic crystalline structure is preferred at high temperature, whereas a mesomorphic highly disordered phase is mainly obtained at lower temperatures. ${ }^{7,8}$

Several attempts have been made to describe nonisothermal crystallization kinetics with simplifying assumptions $^{9-13}$ and procedures have also been developed to determine the relevant rate parameters with no concern on the experimental conditions encountered during processing where drastic solidification conditions are determined by large pressures, stresses, and temperature gradients. ${ }^{9,10}$ As a matter of fact, the data obtained from traditional techniques, such as calorimetric cooling ramps, are restricted to few degrees Celsius per second. Such cooling rates are orders of magnitude lower than those experienced by the material during polymer processing. In recent years, our group undertook quantitative studies of crystallization under high cooling rates (continuous cooling transformation, $\mathrm{CCT}^{14}$ ) with reference to different materials, such as poly(ethylene terephthalate) $(\mathrm{PET})^{15}$ and polyamide6 (PA6). ${ }^{16,17}$

Furthermore, the crystalline structure of iPP quenched from the melt is affected not only by cooling rate, or generally by processing conditions, but also by molecular parameters like molecular mass $\left(M_{w}\right)$ and molecular mass distribution (MWD). Different configurations (isotacticity and head-to-tail sequences) or addi- 
tion of small monomeric units and nucleating agents can also influence the final structure. ${ }^{18-25}$

Influence of molecular weight on polymer crystallization is controversial. Stem length indeed interferes with entanglement density, thus determining a rate-controlled segregation regime of topological constraints in noncrystalline regions. Very low molecular weight tails of the distribution are shown to positively affect crystallization kinetics, although their thermodynamic action should not favor perfection of crystallites.

It is known from the literature that crystallization kinetics of semicrystalline polymers is influenced by the presence of contaminants. The main effect of the addition of a nucleating agent is an increase of the final crystallinity level together with a higher final density and a finer and homogeneous crystal size distribution. This typical effect of enhancement of the overall crystallization kinetics allows one to infer that crystallization kinetics is nucleation-controlled, being the nucleation step the rate-determining one, while the growth rate remains almost unaffected. ${ }^{22,23}$

On the other hand, the incorporation of a small content of ethylene units in the polypropylene chains has an influence on the regularity of the molecular structure. In fact, a change in tacticity induced by the shortening of isotactic sequences was observed. ${ }^{26}$ Although this has a negative influence on crystallization kinetics, an opposite effect should come from the enhanced mobility due to the presence of the ethylene sequences. As a result of these counteracting effects, a relatively narrow window of cooling rates exists in which an enhancement of crystallization kinetics sets in. ${ }^{19}$

A better understanding of the relation between processing and properties can be achieved if the absolute crystallinity during transformation can be predicted as a function of processing conditions. This prediction has to be supported by a crystallization kinetics model; in this article, a modified two-phase nonisothermal form of the Kolmogoroff-Avrami-Evans model was used to describe the crystallization kinetics. ${ }^{27-32}$

The main purpose of this study is to underline the relevance of thermal history resulting from various cooling conditions on the crystallization kinetics of different grades of iPP containing various additives such as nucleating agents and small content of ethylene.

More specifically, the article attempts to identify relevant material parameters determining quiescent nonisothermal crystallization kinetics simulating polymer solidification under processing conditions. One has obviously to cope with commercially relevant grades, which implies constraints in the span they cover. Therefore limitations arise not only due to the intrinsic poor significance of material parameters to crystallization kinetics but also owing to the limitation on the grades one can recover on the market.

Finally, one of the main issues of this work is the appropriate comparison among the investigated iPP samples to outline, when possible, the influence on the crystallization kinetics of average molecular mass, molecular-mass distribution, isotacticity, copolymerization with small amount of ethylene units, and the addition of nucleating agents.

\section{THEORY: CRYSTALLIZATION KINETICS MODEL}

When dealing with crystallization of iPP, the numerous crystalline modifications of this material must be accounted for, since $\alpha, \beta$, or $\gamma$ crystals may form upon solidification from the melt. The resulting complex frame can be simplified based on some experimental evidences, supported by several references. ${ }^{19,22-24}$ As for the $\beta$ phase, it basically shows up only if specific $\beta$ nucleants are added; therefore, for commercial non- $\beta$ nucleated iPPs, it does not form ${ }^{22-24}$; traces of $\gamma$ form crystals are often present, but always in minor amount and in a narrow window of operating conditions (i.e., cooling rates), hence its presence is neglected without affecting the reliability of the results. ${ }^{19}$

Under the aforementioned hypotheses, as two different crystalline phases are formed ( $\alpha$ and mesomorphic), at least two kinetic processes take place simultaneously. The simplest model is a parallel of two kinetic processes noninteracting and competing for the available molten material. The kinetic equation adopted here for both processes is the nonisothermal formulation by Nakamura et al., ${ }^{90}$ of the KolmogoroffAvrami-Evans model. ${ }^{27-30}$

The model is based on the following equation:

$$
\frac{X(t)}{X_{\infty}}=1-\exp [-E(t)]
$$

where $X(t)$ and $X_{\infty}$ are the crystallized volume fraction at time $t$ and in equilibrium conditions, respectively. For simplicity and for the sake of generalization, $X_{\infty}$ is here assumed to be a material constant, although it has been reported its dependence upon the crystallization history (crystal size distribution and degree of perfection). ${ }^{33}$

$E(t)$ is the expectancy of crystallized volume fraction if no impingement would occur. A different formulation of the model can be easily obtained by differentiation of eq. (1), thus getting

$$
\frac{d \xi}{d t}=(1-\xi) \dot{E}(t)
$$

where the relative volume fraction is

$$
\xi=\frac{X(t)}{X_{\infty}}
$$

Since in the case of interest two crystalline phases develop, the simplest extension of the present model is to assume that those phases grow independently in 
parallel, competing each other for the residual fraction of available melt. Under this hypothesis, the rate equation, for the general case of $m$ crystalline phases developing simultaneously, becomes

$$
\frac{d \xi_{i}}{d t}=\left(1-\sum_{i} \xi_{i}\right) \dot{E}_{i}(t) \quad \text { for } i=1, \ldots, m
$$

The following function, suggested by several authors, ${ }^{31-34}$ can be adopted for the expression of the time derivative of the expectancy, leading to a rate equation proportional to the fraction of untransformed material times the current value of the kinetic constant, in which nucleation and growth rates have been lumped together (nucleation and growth are therefore isokinetic):

$$
\begin{array}{r}
\frac{d \xi_{i}}{d t}=\left(1-\sum_{i} \xi_{i}\right) n_{i} \ln 2\left[\int_{0}^{t} K_{i}(T) d s\right]^{n_{i}-1} K_{i}(T) \\
i=1, \ldots, m
\end{array}
$$

The form adopted in eq. (5) for the time derivative of the expectancy reduces to the classical Avrami form, with a dimensionality index $n_{i}$ for the $i$ th phase, if an isothermal experiment is considered. As for the dependence of the rate constant $K_{i}$ on temperature, the simplest expression that one can consider is a Gaussian shaped curve:

$K_{i}(T)=K_{0, i} \exp \left[-\frac{4 \ln 2\left(T-T_{\max , i}\right)^{2}}{D_{i}^{2}}\right] \quad i=1, \ldots, m$

where $D_{i}, T_{\max , i}$ and $K_{0, i}$ are the half width, the temperature where the maximum of $K_{i}$ is attained, and the maximum value of $K_{i}$ itself, respectively. ${ }^{33}$

The governing equations with reference to two phases ( $\alpha$ and mesomorphic phase) are the following:

$$
\begin{gathered}
\frac{d \xi_{\alpha}}{d t}=\left(1-\xi_{\alpha}-\xi_{m}\right) n_{\alpha} \ln 2\left[\int_{0}^{t} K_{\alpha}(T) d s\right]^{n_{\alpha}-1} K_{\alpha}(T) \\
\frac{d \xi_{m}}{d t}=\left(1-\xi_{\alpha}-\xi_{m}\right) n_{m} \ln 2\left[\int_{0}^{t} K_{m}(T) d s\right]^{n_{m}-1} K_{m}(T)
\end{gathered}
$$

where $\alpha$ and $m$ indices stand for the monoclinic and the mesomorphic phases, respectively.

This system of two coupled ordinary differential equations can be integrated with the appropriate initial conditions $\left(\xi_{\alpha}=\xi_{m}=0\right.$ for $\left.t=0\right)$. The integration leads to crystallinity development with time under any temperature history.

Figure 1 shows a typical $K(T)$ curve for the two different phases, and Figure 2 outlines the influence of two main parameters, the product of $K_{0}$ and $D$ (nearly the area under the $K(T)$ curve sometimes called crys-

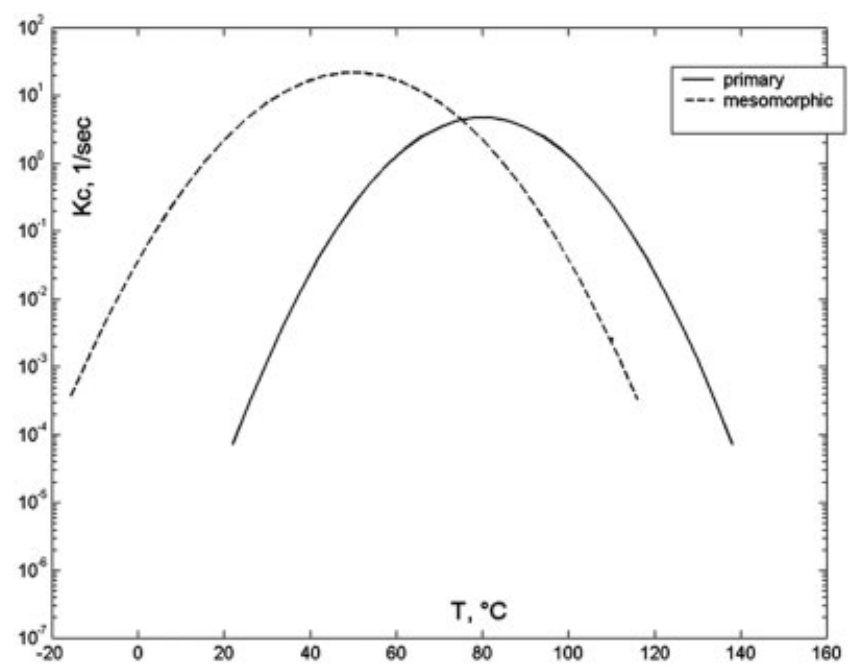

Figure 1 Kinetics constant versus temperature for $\alpha$ and mesomorphic phase.

tallizability) $)^{33}$ and the Avrami index $n$. This latter is representative of the sensitivity of the crystallization kinetics to the cooling rate, a larger $n$ leads in fact to a faster dependence of final crystallinity on cooling rate, the curves crossover is however always the same, i.e., about one half of the maximum attainable crystallization at an abscissa of $K_{0} D$. The crystallizability is a cooling rate scaling factor of crystallization kinetics; as a matter of fact, a larger value of $K_{0} D$ leads to a shift along the abscissa of the curve, i.e., along the cooling rate, such that the larger the crystallizability, the more pronounced the material tendency to crystallize. $^{33}$

Before discussing the results obtained concerning the sensitivity of the cited parameters on polymer composition, it is worth to point out the intrinsic limitations of the approach adopted related to its empirical nature. They depend on the origin of the KAE equation describing the nucleation and growth without diffusivity constraints and without accounting for the possible nonisokinetic contribution of each mechanism, with a simple mathematical extension to the nonisothermal conditions and finally without accounting for the complexity of crystallization in polymer melts, clearly a multistage process. ${ }^{1}$

A slightly different modeling is represented by the so-called "Schneider rate equations"13; Schneider et al. underline that their approach consists in an application of Avrami's (and Tobin's) impingement model leading to a different mathematical and more easy-to-handle formulation, based on a set of differential equations instead of dealing with integral equations. In other words, although their formulation enhances the applicability to process modeling, the physics behind it is completely described by the Avrami model. Therefore, the use of Schneider's 


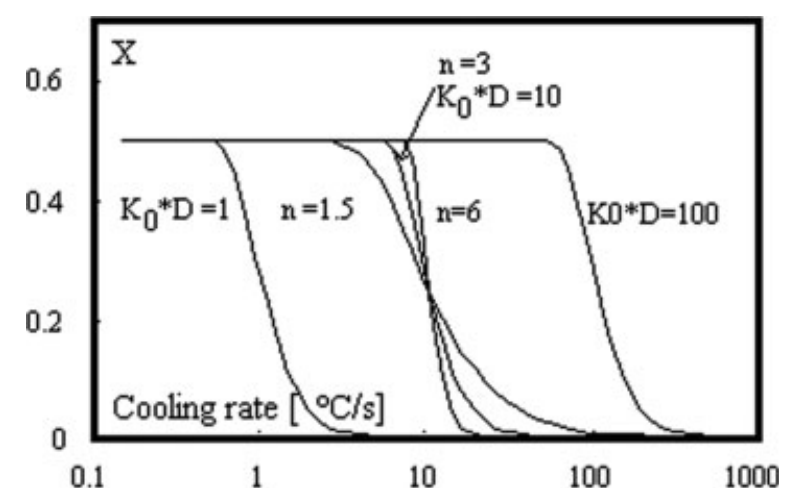

Figure 2 Crystallinity volume fraction as a function of cooling rate.

approach is more advisable when dealing with "nonlumped" problem, to be solved by coupling of transport equations.

All things considered, an analysis of the literature studies on polymer crystallization kinetics shows that the isokinetics approach is the most widely adopted (see the recent review by Pantani et $\mathrm{al}^{35}$ ); moreover, the limitations imposed by the isokinetic hypothesis do not weaken the self consistency and the abundance of information provided here. In any case, the limits of the aforementioned approach can be overcome by recalling the original Kolmogoroff's model (which accounts for the number of nuclei per unit volume on spherulitic growth rate) and determining the average radius of spherulites based on geometrical considerations (i.e., counting the number of nuclei), as shown by Zuidema et al. ${ }^{36}$ and Pantani et al. ${ }^{37}$ This approach has however some limitations, since it can be applied only to conditions where a recognizable spherulitic morphology is formed, i.e., either low cooling rates or conditions where the spherulites are dispersed in a noncrystalline matrix, as in the case of mesomorphic iPP phase. ${ }^{2}$ This possible refinement of the analysis is however far beyond the scope of the present article, both due to its aforementioned limitations and to the macroscopic approach here adopted, aiming to describe crystallization kinetics parameters in the broadest possible range of quiescent solidification conditions, i.e., under conditions emulating polymer processing, except for the role of orientation and pressure.

\section{EXPERIMENTAL}

Different grades of iPP, kindly supplied by Borealis and Himont, were used in this work. The main features of the different grades of iPP tested are listed in Table I.

The iPP samples were subjected to cooling from the melt after being kept at $250^{\circ} \mathrm{C}$ for $30 \mathrm{~min}$ to circumvent memory effects. ${ }^{38,39}$ Samples of each material were prepared under cooling rates ranging from 0.01 to $1000^{\circ} \mathrm{C} / \mathrm{s}$. The experimental procedure, involving also the recording of the thermal history the polymer undergoes during solidification, is extensively described elsewhere. ${ }^{3,14}$ One of the major features of the protocol adopted concerns the issue of sample homogeneity, which is crucial to the method, being the recorded thermal history the only information for determining the final sample structure. Therefore, macroscopic probes such as density and wide-angle X-ray diffraction (WAXD) may be used to characterize the structure attained.

During this kind of experiments, the cooling rates are not constant; the reference cooling rate for each thermal history was chosen as the one experienced by the sample at the iPP characteristic temperature of $70^{\circ} \mathrm{C}^{2,14}$

Figure 3 reports a typical set of cooling histories (ranging from 0.1 up to nearly $1000^{\circ} \mathrm{C} / \mathrm{s}$ ) to which materials were subjected.

Final densities have been determined by a waterethyl alcohol density gradient column kept at $10^{\circ} \mathrm{C}$. Figure 4 reports an example of density versus cooling rate for an iPP grade. In particular, a "pure" iPP (free of additives) is shown. Three zone, characterized by different features, can be distinguished: ${ }^{2,14}$

i. At low cooling rates, the density slowly decreases with cooling rate.

ii. At the highest cooling rates, a low plateau density is observed, related to the onset of the metastable phase of iPP (mesomorphic phase). Once this plateau is reached, density does not change further upon increasing cooling rate.

iii. In an intermediate cooling rate range, the two zones are connected together by a region where the material exhibits a significant density drop upon increasing cooling rate (density cut-off).

TABLE I

Characteristics of the Materials Studied in This Work

\begin{tabular}{|c|c|c|c|c|}
\hline $\begin{array}{l}\text { Material } \\
\text { name }\end{array}$ & $M_{w}$ & MWD & $X_{s}{ }^{\mathrm{a}}$ & Notes \\
\hline НРВ & 430,000 & 6.6 & 2.9 & \\
\hline M2 & 208,000 & 3.5 & 4.5 & \\
\hline M6 & 391,000 & 5.6 & 4.6 & \\
\hline M7N & 379,000 & 5.3 & 3.4 & +Talc 1000 ppm \\
\hline M9 & 380,000 & 3.8 & 5.0 & $\begin{array}{l}\text { Copolymer } \\
0.5 \% \text { ethylene }\end{array}$ \\
\hline M12 & 252,000 & 5.4 & 13.9 & \\
\hline M14 & 293,000 & 7.3 & 5.2 & $\begin{array}{l}\text { Copolymer } \\
3.1 \% \text { ethylene }+ \text { DBS }\end{array}$ \\
\hline M16 & 293,000 & 7.3 & 5.2 & $\begin{array}{l}\text { Copolymer } \\
3.1 \% \text { ethylene }\end{array}$ \\
\hline iPP1 & 476,000 & 6 & & \\
\hline iPP2 & 405,000 & 26 & & Bimodal MWD \\
\hline iPP3 & 489,000 & 9.7 & & \\
\hline iPP4 & 481,000 & 6.4 & & \\
\hline
\end{tabular}

a Xylene soluble weight percentage.

Journal of Applied Polymer Science DOI 10.1002/app 


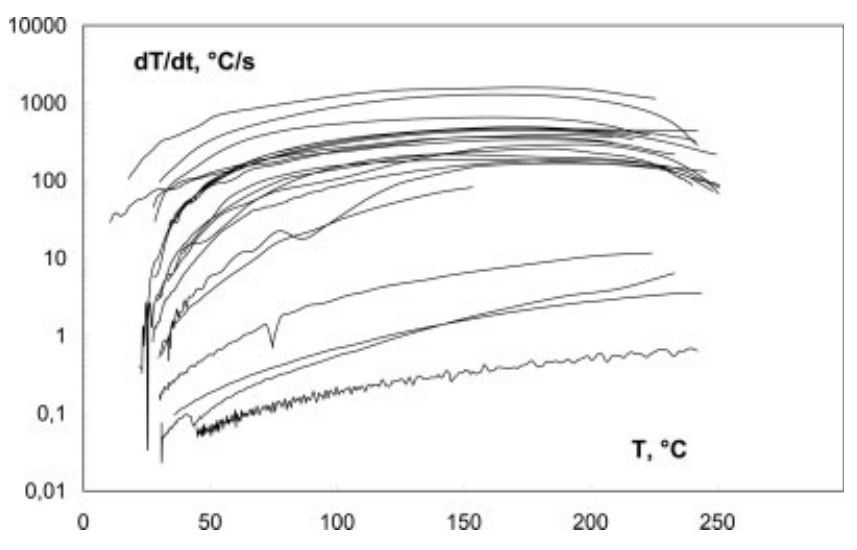

Figure 3 Cooling versus temperature in quenching tests.

Wide angle X-ray diffraction (WAXD) measurements were performed by a Philips diffractometer. The $\mathrm{Cu} \mathrm{K} \alpha$ Nickel-filtered radiation was produced by a Philips PW1150 generator. A computer-assisted goniometer accomplished an optimized step scanning of 0.05 to $0.2^{\circ}$ in the $2 \theta$ interval from $6^{\circ}$ to $35^{\circ}$, depending on the peak height and derivative, with a count time of $60 \mathrm{~s}$.

X-ray patterns, reported in Figure 5, follow the same trend as density with cooling rate, showing a continuous increase of width at half-height of crystalline entities as cooling rate decreases. At low cooling rates stable phases are formed, while at the highest cooling rates the mesomorphic one forms. In an intermediate cooling rate range, the stable monoclinic phase content decreases with cooling rate, while the mesomorphic one increases. ${ }^{2,14}$

The amount of the phases is determined by a deconvolution procedure based on the best fitting of euristic functions to the WAXD experimental data, representa-

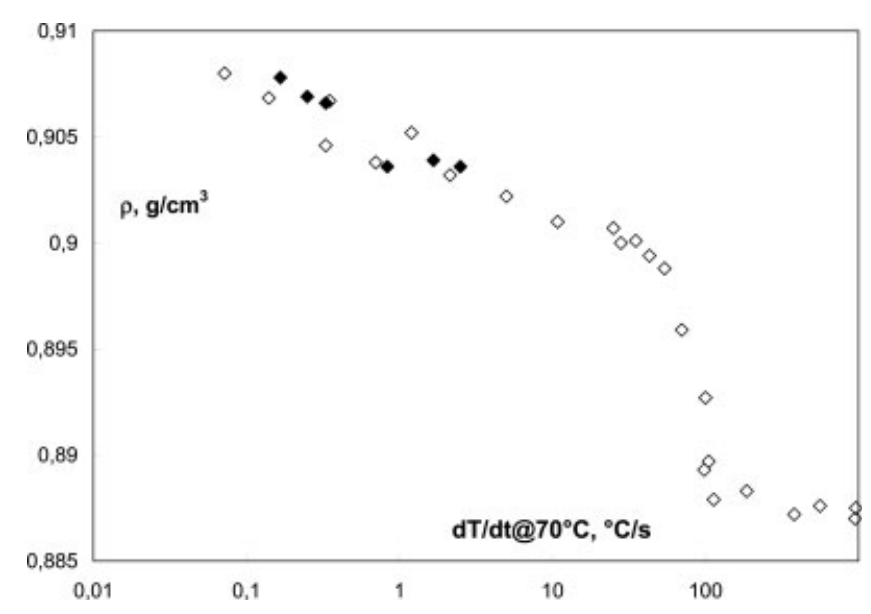

Figure 4 Density versus cooling rate at $70^{\circ} \mathrm{C}$. Filled symbols: samples prepared under constant cooling rate in a DSC apparatus; open symbols: samples prepared in a quenching device. $^{14}$

Journal of Applied Polymer Science DOI 10.1002/app

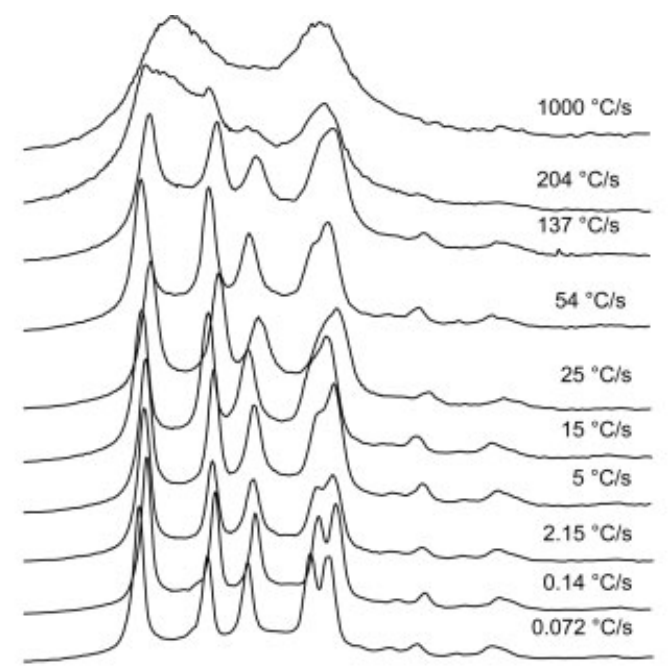

$2 \theta$

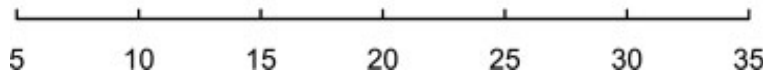

Figure 5 WAXD patterns for a set of samples at different cooling rate.

tive of pure phases spectra. The deconvolution proto$\mathrm{Col}^{40}$ utilizes Pearson VII functions either for the amorphous halo or for the crystalline components, monoclinic and mesomorphic one. In this approach, all the patterns of the same series (a series is referred to the same material quenched at different cooling rates) are simultaneously subjected to the fitting routine. Some of the fitting parameters are globally optimized over the whole experimental pattern, whereas others are locally determined by a single diffraction pattern. The main benefit involved by such procedure is that the samples of the series are subjected to internal comparison by a unique fitting run, so that, even if the amounts of the phases could not have an absolute significance, the relative values are indeed reliable.

A typical deconvolution obtained by this procedure is shown in Figure 6. The monoclinic, mesomorphic,

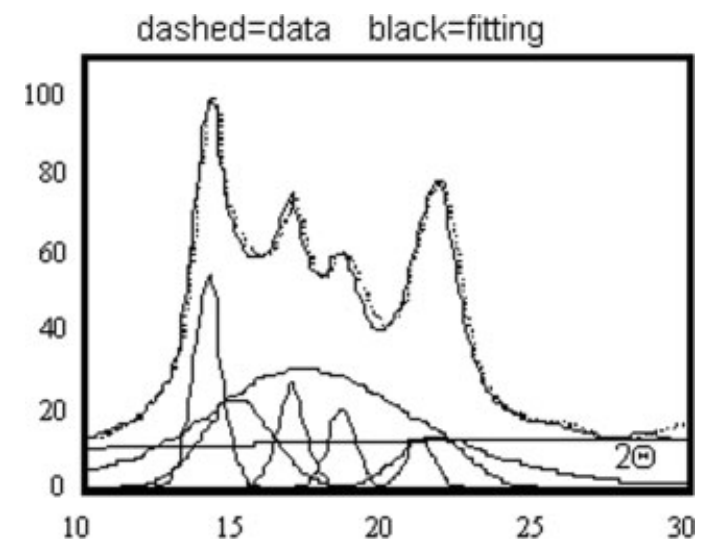

Figure 6 Typical deconvolution of WAXD patterns. 


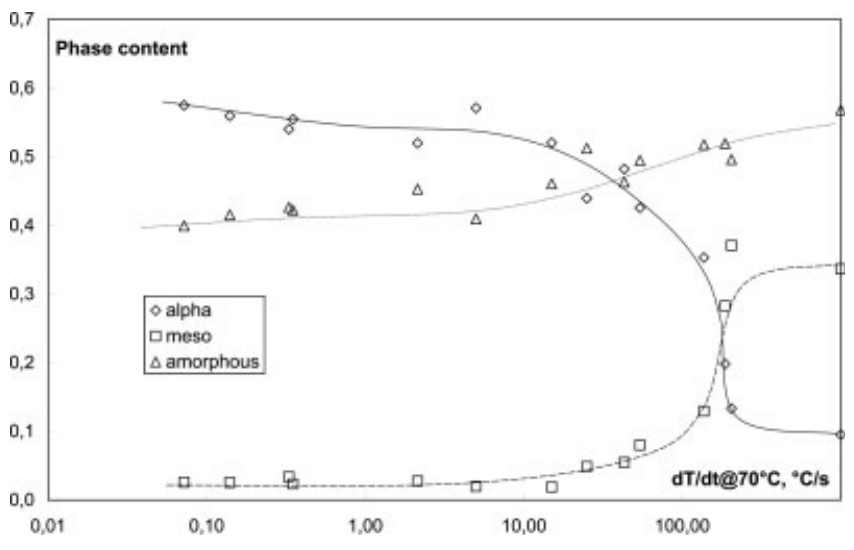

Figure 7 Final crystallinity versus cooling rate.

and amorphous phase amounts are plotted in Figure 7 as function of cooling rate.

The results shown in Figure 7 have been predicted by the authors for an iPP grade in the frame of the KAE approach, including a secondary crystallization in series to the primary at low cooling rates, as shown in a previous article. ${ }^{31}$ Details will not be reported here for the sake of brevity, going beyond the scope of the present article.

In isotactic poly(propylene) (iPP) samples obtained by quenching from the melt, three phases can be simultaneously present:

i. The amorphous phase

ii. The $\alpha$ monoclinic one

iii. The mesomorphic one. ${ }^{41,42}$

The problem of the determination of the amounts of phases is quite complicated particularly when the cooling rates are very high. The mesomorphic phase, in fact, is a disordered phase and it exhibits rather broad diffraction maxima similarly to the amorphous halo.

The availability of a reliable procedure for the determination of the amounts of phases for iPP should allow the physically grounded statement of a constitutive equation for the nonisothermal crystallization

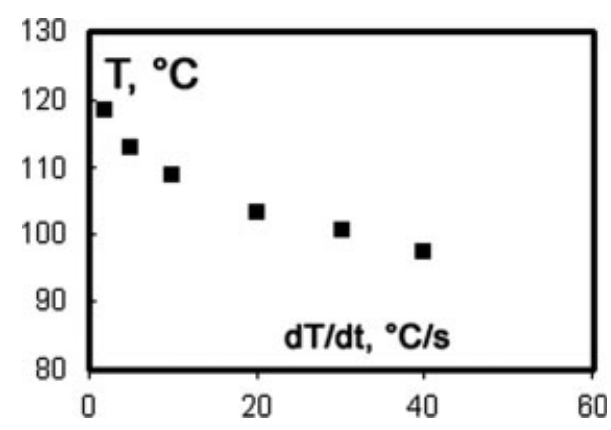

Figure 8 Temperature of maximum crystallization rate from DSC constant cooling experiments.

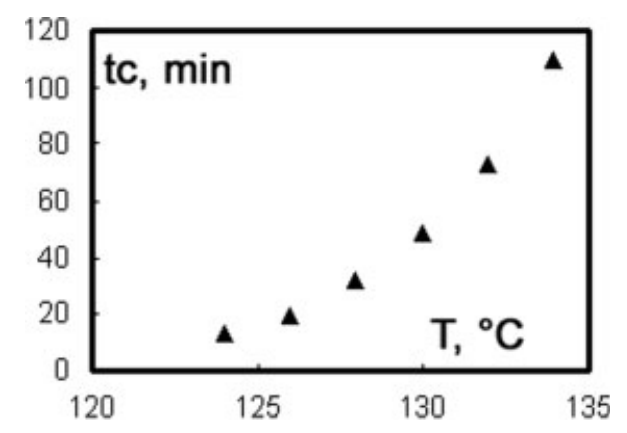

Figure 9 Time of the maximum crystallization rate from isothermal experiments.

behavior, like those recently suggested for multiple phases formation. $3,17,31,32$

DSC tests in the interval $1-40^{\circ} \mathrm{C} / \mathrm{min}$ have been performed in a Perkin-Elmer DSC7 apparatus. In addition, isothermal tests were performed in the same apparatus (Figs. 8 and 9).

The data available for the kinetics description cover a very wide range of crystallization conditions. Sets including calorimetric crystallization isotherms and DSC cooling ramps, as well as quenched samples of known cooling history, were used to identify the crystallization kinetics parameters.

\section{RESULTS AND DISCUSSION: DENSITY DATA AND CRYSTALLIZATION KINETIC MODEL PARAMETERS}

Figures 10-13 show a comparison of the density dependence upon cooling rate for the iPP grades studied, whereas Table II reports the crystallization kinetics model parameters calculated by a best fitting procedure not only on the basis of the final monoclinic and mesomorphic content of the quenched samples, taken from the deconvolution of the WAXD patterns, but also accounting for results that provide the time and the temperature at the maxima of the crystallization rate (isothermal tests and DSC measurements),

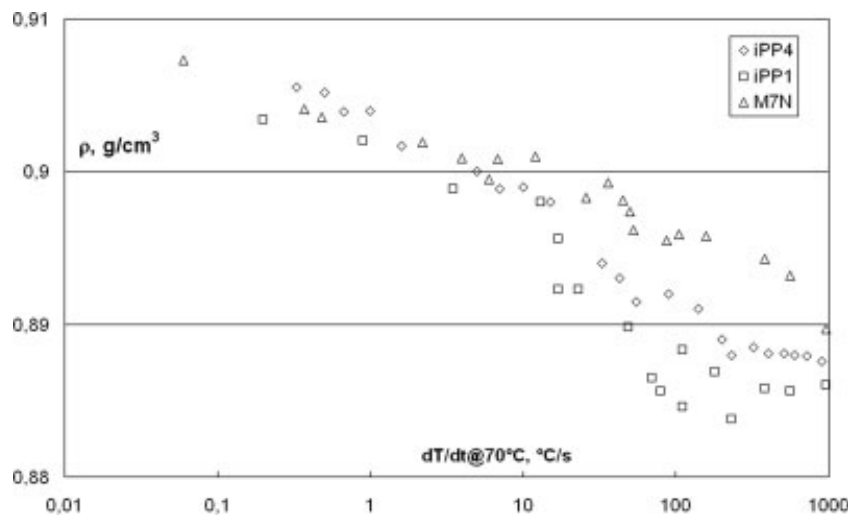

Figure 10 Effect of nucleating agents onto the density versus cooling rate behavior. 


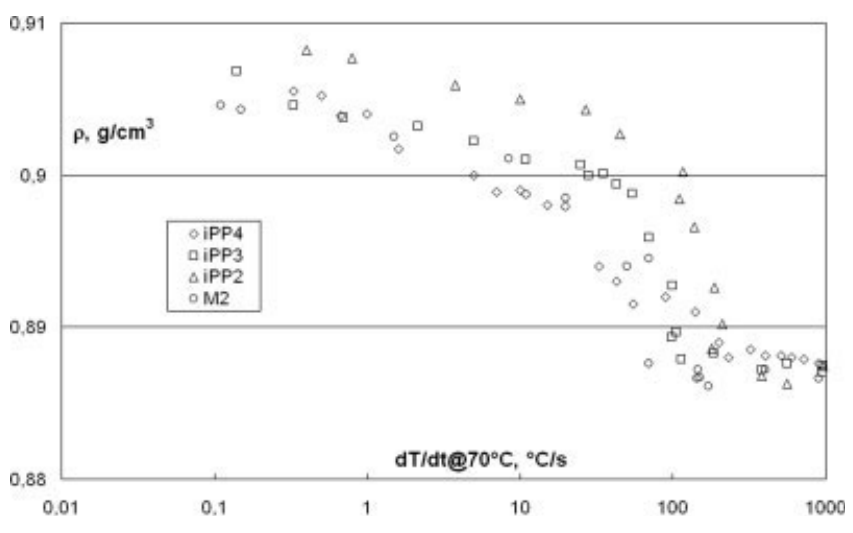

Figure 11 Effect of molecular weight distribution onto the density versus cooling rate behavior.

respectively. For this purpose, a multiobjective optimization code was adopted.

It should be noticed that Table II reports for the mesomorphic phase a range of values both for the Avrami index $n$ and for $X_{\infty}$. The uncertainty in those parameters is however not critical for the purpose of the present work. As a matter of fact, a variability of $n$ between 0.4 and 0.5 reflects into very slight changes in the temperature field in which crystallization takes place; consequently, the influence of this parameter is of minor entity. As for $X_{\infty}$ of the mesomorphic phase, although its variability could turn into larger changes in the crystallization temperature window, its influence is confined to a cooling rate region in which the crystallization of the $\alpha$ phase is very little (very high cooling rates), thus not affecting the $\alpha$ phase kinetic parameters.

As for the Avrami index of the crystalline $\alpha$ phase, Table II reports values equal to 2.0 or 3.0, due to a slight round-off with respect to the results obtained via simulation. The Avrami index is here intended as a mere fitting parameter, in line with most of the literature concerning polymer crystallization kinetics (see for instance the review of Pantani et al. ${ }^{37}$ and the

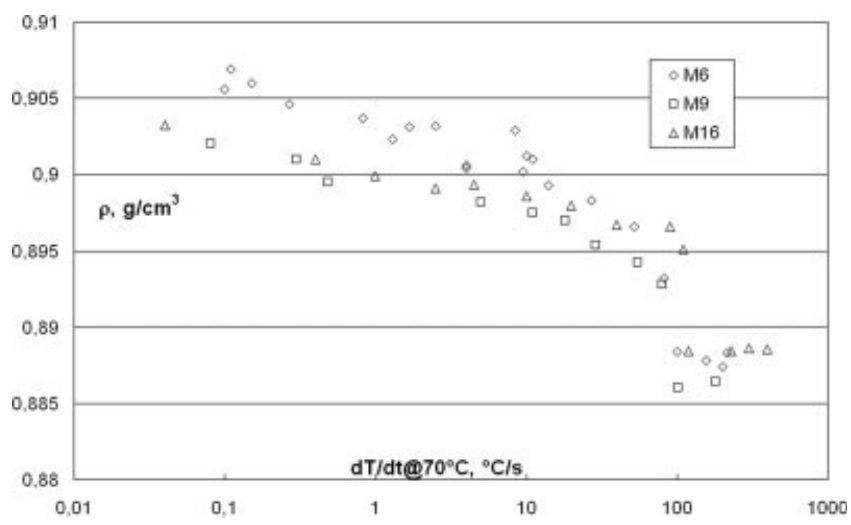

Figure 12 Effect of ethylene content onto the density versus cooling rate behavior.

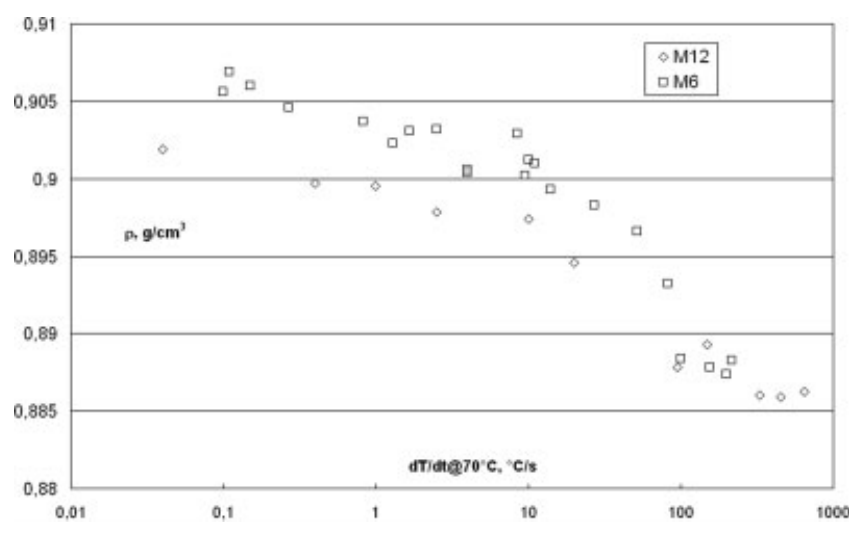

Figure 13 Effect of tacticity onto the density versus cooling rate behavior.

review of Di Lorenzo and Silvestre ${ }^{43}$ ), although its exact physical meaning should indicate the dimensionality of growth (namely three or four for volume filling depending whether nucleation is predetermined of sporadic). In other words, the Avrami index points out only the sensitivity of the crystallization kinetics to the cooling rate, a larger $n$ leads in fact to a faster dependence of final crystallinity on cooling rate, the curves crossover being always the same, i.e., about one half of the maximum attainable crystallization at an abscissa of $K_{0} D$.

Table II shows that differences in materials do not appear to be related in a simple way to kinetic parameters. This may be due to the fact that the set of materials investigated in this work, since representative of iPPs of industrial use, does not cover a wide range of fundamental molecular parameters $M_{w}$ and MWD. As a matter of fact, the limited range of the molecular parameters here explored probably does not comply with a complete enlightenment of the role played by each single factor onto the crystallization behavior.

Nevertheless, some information can be drawn from the table summarizing material kinetics behavior. For example, the so-called "crystallizability," i.e., the product $K_{0} D$ instead of the two separate kinetic parameters, allows one to discuss the differences in the nonisothermal crystallization behavior in relationship to the materials investigated in this work. The crystallizability, roughly corresponding to area under the kinetic constant curve versus temperature, has the dimension of a cooling rate, and indicates somehow the ability of the polymer to crystallize. ${ }^{33}$ A comparison of crystallizability values gives a good insight into the influence of molecular parameters on the crystallization kinetics behavior. For instance, referring to the monoclinic phase only, it can be observed that the smallest value of $K_{0} D$ was obtained for the sample without additives having the highest $M_{w}$ and narrowest MWD. The highest values of crystallizability are however observed for nucleated iPPs (M7N and M14). 
TABLE II

Crystallization Kinetics Parameters

\begin{tabular}{|c|c|c|c|c|c|c|c|c|c|c|c|c|}
\hline \multirow[b]{2}{*}{ Material } & \multicolumn{6}{|c|}{ Monoclinic } & \multicolumn{6}{|c|}{ Mesomorphic } \\
\hline & $\begin{array}{c}K_{0} \\
\left(\mathrm{~s}^{-1}\right)\end{array}$ & $\begin{array}{l}T_{\max } \\
\left({ }^{\circ} \mathrm{C}\right)\end{array}$ & $\begin{array}{c}D \\
\left({ }^{\circ} \mathrm{C}\right)\end{array}$ & $n$ & $X_{\infty}$ & $\begin{array}{c}K_{0} D \\
\left({ }^{\circ} \mathrm{Cs}^{-1}\right)\end{array}$ & $\begin{array}{c}K_{0} \\
\left(s^{-1}\right)\end{array}$ & $\begin{array}{l}T_{\max } \\
\left({ }^{\circ} \mathrm{C}\right)\end{array}$ & $\begin{array}{c}D \\
\left({ }^{\circ} \mathrm{C}\right)\end{array}$ & $n$ & $X_{\infty}$ & $\begin{array}{c}K_{0} D \\
\left({ }^{\circ} \mathrm{Cs}^{-1}\right)\end{array}$ \\
\hline $\mathrm{HPB}$ & 1.6 & 82 & 28 & 2 & 0.55 & 44.8 & 1.6 & 57 & 19 & $0.4-0.5$ & $0.45-0.55$ & 30.4 \\
\hline M2 & 1.4 & 77.3 & 33 & 3.0 & 0.60 & 46.2 & 0.6 & 40 & 30 & $0.4-0.5$ & $0.45-0.55$ & 18 \\
\hline M12 & 2.5 & 79 & 30 & 2.0 & 0.51 & 75 & 3.3 & 42 & 31 & $0.4-0.5$ & $0.45-0.55$ & 94 \\
\hline $\mathrm{M} 7 \mathrm{~N}$ & 8.0 & 82 & 30 & 2 & 0.48 & 240 & NA & NA & NA & $0.4-0.5$ & $0.45-0.55$ & NA \\
\hline M9 & 2.0 & 70 & 36 & 3.0 & 0.53 & 102 & 2.0 & 40 & 36 & $0.4-0.5$ & $0.45-0.55$ & 72 \\
\hline M6 & 2.4 & 66 & 40 & 3.0 & 0.54 & 96 & 2.0 & 40 & 40 & $0.4-0.5$ & $0.45-0.55$ & 80 \\
\hline M14 & 40 & 72 & 29 & 3.0 & 0.40 & 1160 & 1.0 & 40 & 34 & $0.4-0.5$ & $0.45-0.55$ & 34 \\
\hline M16 & 1.8 & 71 & 33 & 3.0 & 0.50 & 99.4 & 1.0 & 40 & 34 & $0.4-0.5$ & $0.45-0.55$ & 34 \\
\hline iPP1 & 1.6 & 82 & 28 & 2 & 0.55 & 44.8 & 1.6 & 57 & 19 & $0.4-0.5$ & $0.45-0.55$ & 30.4 \\
\hline iPP2 & 3.5 & 73 & 34 & 2 & 0.50 & 255.5 & 1.5 & 40 & 31 & $0.4-0.5$ & $0.45-0.55$ & 60 \\
\hline iPP3 & 2.7 & 70 & 35 & 2 & 0.57 & 189 & 0.22 & 40 & 40 & $0.4-0.5$ & $0.45-0.55$ & 8.8 \\
\hline iPP4 & 4.5 & 85 & 27 & 2 & 0.45 & 121 & 0.27 & 53.5 & 33.8 & $0.4-0.5$ & $0.45-0.55$ & 9.12 \\
\hline
\end{tabular}

All things considered, it should be however underlined that differences in crystallizability below a factor 1.3 cannot be considered reliably assessed by the crystallization kinetics method, due to the intrinsic errors in the evaluation of both $K$ and $D$ throughout the best fitting procedure.

If one looks at Figure 10, reporting density as a function of cooling rate for three polymers having similar features (molecular mass and distribution) except for the presence of nucleating agents, one comes to the conclusion that the presence of nucleants shifts the density cut-off toward larger values of cooling rate; as a matter of fact, the calculated crystallizability of the iPP denominated M7N (strongly nucleated) results larger than the one of iPP1 and iPP4.

Higher values of crystallizability are observed when the molecular weight distribution is broader (see for instance materials iPP2 and iPP3). This behavior is clearly shown in Figure 11, where four polymers with MWD ranging from 3.5 (M2) to 26 (iPP2) are reported. The observable shift of density cut-off toward larger cooling rate upon increasing MWD is correctly accompanied by a parallel increase of crystallizability (Table II).

On the other hand, no direct and obvious correlation may be found to relate crystallizability to $M_{w}$. This apparent inconsistency can be reasonably explained by recalling the already mentioned low variability of molecular weights of the iPP grades investigated in this article, related to their "commercial" nature. Consequently, in the light of crystallization behavior, all the iPP molecular weights listed in Table II have to be considered rather similar, being their difference in molecular weight not sufficient to develop dissimilar crystallization kinetics.

Addition of small amounts of ethylene units in the copolymer does not influence significantly any of the kinetic parameters mentioned above, the small changes of the product $K_{0} D$ mainly depending on the differences in MWD and not upon the ethylene content. No significant differences in the product $K_{0} D$ may be argued among materials M6, M9, and M16, although the second couple is copolymerized with ethylene. Also the amount of ethylene used in the copolymerization process does not appear to be relevant. These results are confirmed by density data shown in Figure 12.

On the contrary, the couple of nucleated materials (M7N and M14) that basically differ from the others for the addition of ethylene in the latter show a large difference in the crystallizability, suggesting a synergetic effect of copolymerization with the addition of a nucleating agent on crystallization kinetics. Although the enhancement of chain mobility, which increases with ethylene content, and nucleation are both factors promoting the crystallization kinetics, the source of the synergy is not simple to interpret.

The tacticity index does not seam to have a significant influence on the kinetics of monoclinic $\alpha$ phase. Figure 13 shows that the density cut-off of M12, with a lower tacticity, is slightly anticipated with respect to the one of MP6; on the other hand, crystallizability of M12 is somehow lower than the one of M6 (Table II).

Other kinetics parameters of the monoclinic phase are more difficult to be related to molecular parameters. Additionally, their physical meaning is not straightforward with the exception of Avrami index $n$. This last, in principle, represents the dimensionality of the growth and the kind of nucleation. Experiments, however, rarely well correlate with a value of $n$ in line with the dimensionality of the crystallization process under observation.

Furthermore, the correlation of mesomorphic phase kinetics parameters appears difficult, probably this can be related to the fact that mesomorphic phase determinations are affected by a larger uncertainty due to the broader WAXD peaks characterizing this phase. 
With this respect, some recent cooling experiments performed on a nanocalorimeter ${ }^{44}$ have shown two distinct crystallization peaks ( $\alpha$ and mesomorphic phase) appearing in a quite large range of cooling rates, the crystallization of the $\alpha$ taking place up to about $1000^{\circ} \mathrm{C} / \mathrm{s}$. The apparent contradiction with the results here presented (alpha phase disappearing above $200-300^{\circ} \mathrm{C} / \mathrm{s}$ ) may be consistently solved if one considers that the amount of $\alpha$ phase formed at high cooling rates is of the order of a few percent, hence below the measure limits of WAXD (around 5\%). Secondly, being the sample mass undergoing the DSC cooling run in the nanocalorimeter of the order of a few nanograms, the enhancement of crystallization due to the "surface effect" (high constraints due to the low sample size with respect to the average radius of giration) must be taken into account. Thirdly, the presence of a mesomorphic phase crystallization peak at room temperature justifies the difficulties encountered in iPP amorphization, as confirmed by the present results where a consistent value of the crystallization kinetic constant of the mesomorphic phase at room temperature is shown (Table II).

Finally, if one considers that, with a few exceptions, the study was executed on a set of materials of industrial interest, a conclusion can be drawn about the fact that crystallization kinetics is mainly influenced only by the presence of nucleating agents. The influence of copolymerization on crystallization kinetics being relevant only if coupled with nucleation.

\section{CONCLUSIONS}

A systematic investigation about the crystallization kinetics under cooling rates typical of polymer processing for several commercial iPP grades was carried out, aiming to highlight the relevance of a number of molecular parameters, including molecular weight and distribution, tacticity, ethylene units content, and nucleating agents.

The approach adopted, although the equations used are clearly empirical, is rather general and it surely represents a development with respect to phenomenological procedures describing relationships between structure and processing conditions. In the intention of the article, the kinetic parameters are the connections among such macroscopic observations.

Furthermore, the article provides a large amount of consistent experimental data under nonisothermal conditions (cooling rate range from below 0.1 to above $1000^{\circ} \mathrm{C} / \mathrm{s}$ ) for a broad set of commercial iPPs so far not extensively reported in literature.

It should be however underlined that the model provides values of $K(T)$ comparable for the different grades, $K(T)$ being the reciprocal of half-crystallization isothermal time regardless the value of the Avrami index.
The most influential factor turned out to be the presence of nucleating agents, which shifts toward larger value the material intrinsic "crystallizability" (represented by area under the "bell-shaped" crystallization kinetics constant versus temperature curve). In particular, the effect of molecular weight does not appear to be very relevant, due to the limited range of molecular weights available in material grades of a "commercial" nature.

On the other hand, an increase in the polydispersity index significantly reflects into a parallel increase in crystallizability. Finally, addition of small amounts of ethylene units in the copolymer does not appreciably influence the kinetic parameters unless combined with nucleation.

\section{References}

1. Strobl, G. The Physics of Polymers. Concepts for Understanding Their Structures and Behavior; Springer: New York, 1997.

2. Piccarolo, S. J Macromol Sci Phys 1992, B31, 501.

3. Piccarolo, S.; Saiu, G.; Brucato, V.; Titomanlio, G. J Appl Polym Sci 1992, 46, 625.

4. Choi, C.; White, J. L. Polym Eng Sci 2000, 40, 655.

5. Rotter, G.; Ishida, H. J Polym Sci Part B: Polym Phys 1992, 30, 489.

6. Fu, Y.; Annis, B.; Boller, A.; Jin, Y.; Wunderlich, B. J Polym Sci Part B: Polym Phys 1994, 32, 2289.

7. Paukkeri, R.; Lehtinen, A. Polymer 1993, 34, 4075.

8. Paukkeri, R.; Lehtinen, A. Polymer 1993, 34, 4083.

9. Nakamura, K.; Katayama, K.; Amano, T. J Appl Polym Sci 1973, 17,1031 .

10. Nakamura, K.; Watanabe, T.; Katayama, K.; Amano, T. J Appl Polym Sci 1972, 16, 1077

11. Ziabicki, A. Polymer 1972, 17, 301.

12. Jeziomy, A. Polymer 1978, 19, 1142.

13. Schneider, W.; Koppl, A.; Berger, J. Int Polym Process 1988, 2, 151.

14. Brucato, V.; Piccarolo, S.; La Carrubba, V. Chem Eng Sci 2002, $57,4129$.

15. Piccarolo, S.; Brucato, V.; Kiflie, Z. Polym Eng Sci 2000, 40, 1263.

16. Brucato, V.; Piccarolo, S.; Titomanlio, G. Int J Forming Process $1998,1,35$.

17. Brucato, V.; Crippa, G.; Piccarolo, S.; Titomanlio, G. Polym Eng Sci 1991, 31, 1411.

18. De Rosa, C.; Auriemma, F.; Resconi, L. Macromolecules 2005, 38, 10080.

19. Foresta, T.; Piccarolo, S.; Goldbeck-Wood, G. Polymer 2001, 42, 1167.

20. Sakurai, T.; Nozue, Y.; Kasahara, T.; Mizunuma, K.; Yamaguchi, N.; Tashiro, K.; Amemiya, Y. Polymer 2005, 46, 8846.

21. Chen, J. H.; Tsai, F. C.; Nien, Y. H.; Yeh, P. H. Polymer 2005, 46, 5680.

22. Nagasawa, S.; Fujimori, A.; Masuko, T.; Iguchi, M. Polymer 2005, 46, 5241

23. Raab, M.; Scudla, J.; Kolarik, J. Eur Polym J 2004, 40, 1317.

24. Marigo, A.; Marega, C.; Causin, V.; Ferrari, P. J Appl Polym Sci 2004, 91, 1008 .

25. Elmoumni, A.; Gonzalez-Ruiz, R. A.; Coughlin, E. B.; Winter, H. H. Macromol Chem Phys 2005, 206, 125.

26. Zimmermann, H. J. J Macromol Sci Phys 1993, B32, 141.

27. Avrami, M. J Chem Phys 1939, 7, 1103.

28. Avrami, M. J Chem Phys 1940, 8, 212.

29. Avrami, M. J Chem Phys, 1941, 9, 177.

30. Evans, U. R. Trans Faraday Soc 1945, 41, 365. 
31. La Carrubba, V.; Brucato, V.; Piccarolo, S. J Polym Sci Part B: Polym Phys 2002, 40, 153.

32. Brucato, V.; Piccarolo, S.; Titomanlio, G. Makromol Chem Macromol Symp 1993, 68, 245.

33. Ziabicki, A. Fundamentals of Fibre Formation; Wiley: London, 1976.

34. La Carrubba, V. Polymer Solidification under Pressure and High Cooling Rate, Ph.D. Thesis; CUES: Salerno; 2001. ISBN: 8887030-27-8.

35. Pantani, R.; Coccorullo, I.; Speranza, V.; Titomanlio, G. Prog Polym Sci 2005, 30, 1185.

36. Zuidema, H.; Peter, W. M. P.; Meijer, H. E. H. Macromol Theory Simul 2001, 10, 447.
37. Pantani, R.; Speranza, V.; Coccorullo, I.; Titomanlio, G. Macromol Symp 2002, 185, 309.

38. Ziabicki, A.; Alfonso, G. C. Colloid Polym Sci 1994, 272, 1027.

39. Alfonso, G. C.; Ziabicki, A. Colloid Polym Sci 1995, 273, 317.

40. Martorana, A.; Piccarolo, S.; Scichilone, F. Macromol Chem Phys 1997, 198, 597

41. Turner Jones, A.; Aizlewood, A. M.; Beckett, D. R. Makromol Chem 1964, 75, 134.

42. Corradini, P.; Petraccone, V.; De Rosa, C.; Guerra, G. Macromolecules 1986, 19, 2699.

43. Di Lorenzo, M. L.; Silvestre, C. Prog Polym Sci 1999, 24, 917.

44. De Santis, F. Personal communication, 2005. 